\title{
How Children Use Active Videogames and the Association Between Screen Time and Physical Activity
}

\author{
Cuisle Forde, PhD, and Juliette Hussey, PhD
}

\begin{abstract}
Objective: The energy required to play active videogames (AVGs) has been reported on in the literature; however, little is known about how children use such games in their home environment. The aim of this study was to investigate children's use of AVGs and the association among AVG use, other screen-based activities, and physical activity levels.

Materials and Methods: Eight hundred and twenty children 12.1 (0.6) years of age participated. Physical activity levels, sedentary screen-based activities, and AVG use were investigated. Differences across genders and deprivation indices were also analyzed.

Results: Fifty-eight percent of children met minimal physical activity guidelines. Forty-seven percent of children exceeded screen time recommendations. Of those who had access to AVGs, more children played sedentary games (or active games in a sedentary manner [68 percent]) than active games (55 percent) on AVG consoles. Furthermore, sedentary games were played for longer than active games. AVG play was positively correlated with reported time spent watching television $(P=0.02)$.

Conclusions: In free-living conditions AVG consoles are being used by more children and for longer durations as sedentary screen-based devices rather than active screen-based devices.
\end{abstract}

\section{Introduction}

$\mathbf{R}$ ESEARCH ON THE LEISURE-TIME activities of children has revealed that many use computers and play videogames on a daily basis, some for several hours a day. ${ }^{1,2}$ The American Academy of Pediatrics Committee on Public Education recommend that children should spend no more than 2 hours/day on screen-based activities. ${ }^{3}$ Furthermore, many children are not achieving the recommended 60 minutes of physical activity/day. ${ }^{4,5}$ Traditionally, screenbased activities have been considered sedentary; however, laboratory-based studies have shown that active videogames (AVGs) can be considered a form of physical activity. ${ }^{6}$ Despite these encouraging results, interventional studies conducted in free-living situations with the aim of using AVGs to benefit health have yielded unclear or mixed outcomes. ${ }^{6-8}$ Furthermore, despite high AVG sales, ${ }^{9,10}$ it is not known how many children have access to AVGs, how often they are used, and whether the games are typically played in an active manner or not. Although AVG consoles enable players to be physically active and have the potential to result in moderate-intensity physical activity in youth and adults, ${ }^{11,12}$ sedentary games are also available for these "active" consoles. When children play sedentary games on an AVG console, the activity is analogous to playing a traditional sedentary electronic videogame console. ${ }^{13}$ It is also possible to play AVGs incorrectly with the result that these games become less active or sedentary, for example, playing "Wii "TM Boxing" (Nintendo, Kyoto, Japan) in a seated position using quick movements of the wrist instead of punching with the arms.

The lack of specific knowledge on children's use of AVGs highlights the infancy of this area and the need for further investigation into the extent to which AVGs feature in children's leisure time and how AVG use relates to other common leisure-time activities, in particular, physical activity and sedentary screen time.

The aim of this study was to investigate screen-based leisure-time activities and physical activity levels of children in Dublin, Ireland. An emphasis was placed on AVG use. Objectives were to determine (1) the percentage of children in sixth class (approximately 12 years old) in Dublin who had access to electronic game consoles, including AVGs, (2) whether children who used AVG consoles played active or sedentary games on those consoles, (3) the associations among AVG play, physical activity levels, and time spent on other screen-based activities and (4) to investigate differences across genders and areas of deprivation. 


\section{Materials and Methods}

A cross-sectional questionnaire design was selected for this study. Ethics approval was granted from the Trinity College Faculty of Health Sciences Ethics Committee. Written informed parental consent and child assent were obtained prior to data being collected. A sample size of several hundred children was sought; therefore, direct measurement was not feasible. The questionnaire used had three sections. The first section asked children to quantify their physical activity levels. The second section asked children to recall time spent using screen-based devices. Finally, the third section asked questions specific to AVG access and use. As this was the first study to examine the typical use of AVGs, questions specific to AVG use had to be developed. To ensure the newly developed questions were appropriate and effective, guidelines on developing questions for children were followed. ${ }^{14-16}$ In the initial stages of developing the questionnaire used in this study, two researchers in the area reviewed it and suggested amendments. For questions relating to physical activity levels and sedentary screen time, established psychometrically sound questionnaires gathering this information were reviewed, and questions considered appropriate to the research question at hand were chosen.

To assess physical activity, children were asked to recall details about any regular physical activities performed in the preceding year. This question was based on Question 4 of the Modified Activity Questionnaire for Adolescents, which has been shown to be valid and reliable ${ }^{17}$ and has been used in studies involving similar populations..$^{5}$ The question asked children to recall activities they carried out at least 10 times in the last year. A list of activities was given that children could tick; there was also space for children to add other activities. For each activity children then estimated, as best they could, how many times a month, how many times a week, and for how long they carried out each activity. This was completed in a grid format, with an example on how to complete the grid given. Because children's activities are known to vary between weekdays and weekends, ${ }^{4,18}$ children were asked to recall certain activities, such as television viewing, over the 7 days preceding completion of the questionnaire. Screen-based activities assessed were television viewing, electronic game use, time spent using social networking sites, and nongaming leisure-time computer use such as browsing on the Internet or typing (the latter category included screen time related to school work). Different choice categories were given for different activities to enable an appropriate amount of choices for each screen-based device while being able to capture, with some sensitivity, a picture of how long children spend using the devices. For example, because it is known that generally children spend longer watching television than they do playing screen-based games, to use the same choice categories for both would likely result in a ceiling or floor effect.

During pilot work children were asked to comment on the appropriateness of the time categories and number of choices given. Results from this pilot work contributed to the development of choice categories.

To determine whether AVGs are played, and played correctly (i.e., actively), on AVG consoles, participants were asked to report on the amount of time in the preceding 7 days that games were played on AVG consoles in an active manner and in a sedentary manner. Games were considered to be active if the child played them while standing. Games were considered sedentary if played seated.

The questionnaire was piloted on nine children. In the pilot study a modified think aloud technique was used. ${ }^{19}$ Retrospective probing was then used to elicit feedback from participants about the questionnaire and the process of completion. Following this exercise, appropriate changes were made to the questionnaire. The questionnaire was designed to be completed by children under adult supervision. As such, children were asked to complete questionnaires under the supervision of a teacher or at home under the supervision of a parent. To reduce any possible disturbances to the school curriculum, the choice of whether questionnaires were completed in the classroom or at home was at the discretion of each school. Parents and teachers were not trained in questionnaire administration but were asked to help when needed in the explanation of questions and not to attempt to provide answers. Pilot work carried out concluded that children in sixth class would be able to complete the questionnaire with little supervision. Confidentiality was maintained by providing clear instructions on the front of the questionnaire as well as the information leaflet that children were not to write their name on the questionnaire.

Children in sixth class from 10 percent of Dublin National Primary Schools were recruited. To ensure proportional representation, a clustered stratified sampling method was used whereby schools were selected, and all sixth class children in selected schools were sampled. A list of all national primary schools in Dublin, excluding those for children with special needs, was obtained from the Irish Department of Education and Skills Web site (www.education.ie). As there are known differences in physical activity levels between socioeconomic classes, schools were grouped first by electoral district and then by deprivation index. ${ }^{20}$ The deprivation index of an area was determined based on four variables: unemployment, low social class, local authority housing, and no car. This information was gathered from the national census. (Please refer to www.sahru.tcd.ie for more information.) Every 10th school on this list was sampled. This method enabled both a geographically and socioeconomically representative group of children in Dublin to be sampled.

Average metabolic equivalent (MET) hours per week for each participant were calculated using information on time spent in physical activities collected and the compendium of physical activities for children. ${ }^{21}$ Associations involving non-normally distributed or ordinal data were carried out using the Spearman rank test. The difference between paired ordinal data was assessed using the Wilcoxon Signed Rank test. Differences between independent sets of data were assessed using the Mann-Whitney U test or the Pearson chisquared test where appropriate. For all analyses, alpha $<0.05$ was considered significant. Descriptive results were reported as percentage of valid answers to the nearest percent.

\section{Results}

There were, in total, 432 eligible schools in the Dublin area. Eighty-five of these schools were approached, and 47 agreed to take part. This represented a participation rate of 55 percent of schools approached. In total, 820 questionnaires were received by the Principal Investigator. The number of 
children in sixth class in schools varied from 2 to 120 . The average response rate was 65 percent. The mean age of participants was $12.1(0.6)$ years. Four hundred four boys and 412 girls responded; 4 children did not specify their sex. All rates of missing data (mean, 1.6 percent; highest, 4.6 percent) were considered acceptably low. ${ }^{22}$ The internal validity of the questionnaire was examined (similar questions were posed, and answers were compared for consistency) and considered acceptable, ${ }^{22}$ with one exception; children were asked to report on the time spent playing games on AVG consoles while standing up as well as while in a seated position. Both questions referred to AVG console use only. Despite pilot work and instructions provided, approximately 15 percent of children reported not having access to an AVG console for the question involving standing AVG play, while only 8 percent of children reported not having access to an AVG console for the question on seated AVG play. Because the response rates for these questions were similar, this discrepancy cannot be attributed to differences in response rates. In the analysis of these questions, those who reported having access to an AVG console in one question and not in the other were excluded from analysis.

\section{Physical activity levels}

Regular physical activities reported per week was a median of 34 MET hours, with a 25th percentile of 15 MET hours and a 75th percentile of 70 MET hours. Children are recommended to accumulate 60 minutes of moderate physical activity/day. ${ }^{23}$ If an intensity of 4 METs is used to represent moderate-intensity physical activity, this is analogous to 28 MET hours/week. Four hundred fifty-two children (58 percent of all participants) met the guideline of 28 MET hours/week.

\section{Time spent using screen-based devices}

Reported time spent by children watching television, playing games on screen-based electronic devices, and using social networking sites is detailed in Table 1. Children were also asked how much time was spent on computers excluding time spent playing computer games, doing school work, or on social networking sites (for example, time spent browsing on the Internet or typing was included in this question). Results are reported in Table 1.

Forty-seven percent of participants $(n=382)$ reported spending an accumulated time of 14 hours or more engaging in screen-based activities during the 7 days preceding completion of the questionnaire. Significantly more boys ( $n=195 ; 48$ percent) than girls $(n=154 ; 37$ percent) reported exceeding screen-time recommendations (chi-squared $P=0.01)$. No difference between genders was seen in any other variable measured. A quarter of participants reported spending over 14 hours/week watching television. Approximately 9 percent of participants exceeded screen time recommendations through electronic game use alone, whereas 4 percent exceeded recommendations using social networking sites alone and 5 percent using computers. There was no difference between those who accumulated greater than or less than 14 hours of screen-based activity/week in the number of AVG consoles they had access to, the time they spent playing games on AVG consoles, or deprivation
Table 1. Reported Time Spent Using Screen-Based Devices in the 7 Days Preceding Completion OF THE QUESTIONNAIRE

Screen-based activity, answer $\quad \mathrm{n}$ (valid percent)

Watching television

$\begin{array}{lc}\text { None } & 43(6) \\ \text { Less than } 5 \text { hours } & 166(21) \\ 5-10 \text { hours } & 260(32) \\ 10-14 \text { hours } & 137(17) \\ 14-20 \text { hours } & 137(17) \\ \text { Over 20 hours } & 67(8) \\ \quad \text { Total } & 810(100)\end{array}$

Playing screen-based electronic games None

Less than 1 hour

$125(15)$

$1-2$ hours

$109(13)$

$115(14)$

2-4 hours

4-6 hours

131 (16)

$100(12)$

6-10 hours

114 (14)

10-14 hours

$50(6)$

Over 14 hours

69 (9)

Total

$813(100)$

Using social networking sites

None

Less than 1 hour

345 (42)

Between 1 and 2 hours

$124(15)$

109 (13)

2-4 hours

90 (11)

4-6 hours

58 (7)

6-10 hours

$41(5)$

10-14 hours

$16(2)$

14-20 hours

$12(2)$

Over 20 hours

21 (3)

Total

$816(100)$

Using a computer (other than playing games, doing

schoolwork, and social networking)

None

Less than 1 hour

$191(24)$

1-2 hours

2-4 hours

4-6 hours

$194(24)$

$156(19)$

$106(13)$

59 (7)

6-10 hours

$42(5)$

10-14 hours

$22(3)$

Over 14 hours

37 (5)

Total

$807(100)$

Results are reported to the nearest valid percent.

index. The average proportion each screen-based activity contributed to total screen time is represented in Figure 1.

\section{Results of questions specific to AVG use}

Ninety-three percent of participants had access to at least one AVG console. Forty-two percent of participants who had access to AVG consoles reported not having played AVGs in an active manner (standing up) in the preceding 7 days (Table 2). A Wilcoxon Signed Rank test revealed a significant difference $(z=-6.543, P<0.001)$ between time spent sitting playing AVGs and standing playing AVGs. The mean of the ranks in favor of seated play was 250 , whereas the mean of the rank in favor of active play was 186, confirming that children spent a significantly longer amount of time playing AVGs in a seated position than in a standing 


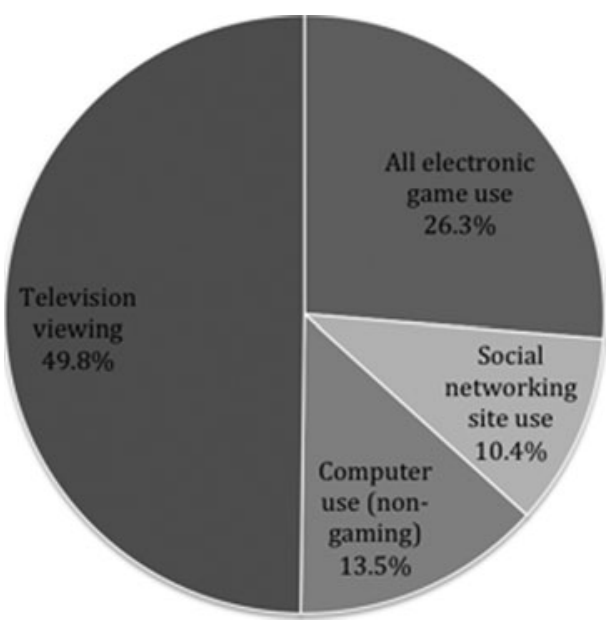

FIG. 1. Average proportion of time each screen-based activity contributed to total screen time per week.

position. Furthermore, more children played sedentary games on AVG consoles ( 73 percent of those who had access to an AVG console) than active games on AVG consoles (58 percent of those who had access to an AVG console).

\section{Association between physical activity and sedentary behavior}

There was a significant negative correlation between MET hours of physical activity per week and both time spent watching television $(\rho=-0.76, P=0.03)$ and time spent using a computer for nongaming purposes $(\rho=-0.08$, $P=0.03$ ). Correlation analysis also showed that MET hours per week were significantly negatively associated with total screen time $(\rho=-0.10, P<0.01)$. There was no correlation between MET hours of physical activity per week and time

Table 2. Response to Questions Regarding How Children Played with Active Videogame Consoles

\begin{tabular}{lcc}
\hline & $\begin{array}{c}\text { Time spent playing on } \\
\text { an AVG console }\end{array}$ \\
\cline { 2 - 3 } & $\begin{array}{c}\text { Active games } \\
\text { (standing } \\
\text { position) }\end{array}$ & $\begin{array}{c}\text { Nonactive } \\
\text { game (seated } \\
\text { position) }\end{array}$ \\
Answer & $50(7)$ & $50(7)$ \\
\hline I do not have an AVG console. & $268(38)$ & $179(25)$ \\
I have an AVG console but & & \\
$\quad$ did not play with it & & \\
in the last 7 days. & $47(7)$ & $61(9)$ \\
Less than 10 minutes & $94(13)$ & $96(14)$ \\
10-30 minutes & $78(11)$ & $74(11)$ \\
30-60 minutes & $88(12)$ & $76(11)$ \\
1-2 hours & $37(5)$ & $70(10)$ \\
2-4 hours & $31(4)$ & $47(7)$ \\
4-7 hours & $14(2)$ & $35(5)$ \\
7-14 hours & $3(<1)$ & $22(3)$ \\
Over 14 hours & $710(100)$ & $710(100)$ \\
$\quad$ Total &
\end{tabular}

Data are number of participants who answered these questions consistently (valid percent).

AVG, active videogame. spent playing AVGs actively. However, a significant association was found between MET hours of physical activity per week and time spent playing AVGs while sitting down $(\rho=0.12, P<0.01)$. Those who achieved the guideline of 28 MET hours/week spent less time engaged in screen-based activities $(\mathrm{U}=70322, P=0.04)$. Those who exceeded screen time recommendations expended significantly fewer MET hours per week $(P=0.04)$. Analysis revealed a significant positive correlation between reported time spent watching television and reported time playing AVGs in both a standing position $(\rho=0.09, P=0.02)$ and a seated position $(\rho=0.18$, $P<0.001)$.

\section{Differences between levels of deprivation}

There was a significant positive correlation between reported time spent on social networking sites and level of deprivation $(\rho=0.123, P<0.001)$, indicating that those in areas of higher deprivation spent longer on social networking sites.

\section{Discussion}

This was the first study to specifically examine the regular use of AVGs and to compare AVG use with other screenbased activities and with physical activity levels. The fact that over 40 percent of children were not achieving physical activity recommendations is a cause for concern. These results are similar to previous research on the physical activity levels across the world, including in Ireland and the United States. ${ }^{24-26}$ Similar to other studies, ${ }^{1,27}$ data from this study have shown that television viewing is the largest contributor to screen time for children in sixth class in Dublin (Table 1). Because there is evidence that television viewing is not only a sedentary behavior but is associated with unhealthy snacking behavior, ${ }^{28}$ efforts to reduce sedentary behavior among children, through either the use of AVGs or other activities, should concentrate on displacing time spent watching television. Forty-seven percent of participants accumulated a total screen time of greater than 14 hours/week. Regardless of activity levels, sedentary activity is known to be associated with negative health effects ${ }^{29}$; therefore this is a matter of concern in itself. Of greater concern is that those who accumulated more screen time engaged in significantly fewer MET hours of physical activity per week. This trend has been reported before ${ }^{27}$ and highlights the existence of a sedentary inactive cohort of youth who may be at risk of developing health problems, including the metabolic syndrome. ${ }^{30,31}$

An objective of this study was to determine the percentage of children in sixth class in Dublin who have access to electronic game consoles, including AVG consoles. Results show that exposure to AVG consoles is widespread among children in sixth class in Dublin. Only 7 percent of participants did not have access to an AVG console. Although AVG consoles are widely available to children, 42 percent of participants with access to an AVG console reported not having played it in the 7 days preceding completion of this questionnaire. Another objective of this study was to determine whether children who use AVG consoles play active or sedentary games. Among those who did report playing with AVG consoles, there is evidence to suggest that few are gaining exercise through AVG use because significantly 
more children reported having used them to play sedentary games than active games.

One of the ways AVG play could theoretically benefit health would be to replace more sedentary screen-based activities such as television viewing. ${ }^{32}$ The results of this study found positive correlations between time spent watching television and time spent playing AVGs in both an active and sedentary manner, suggesting that those who spend time playing AVGs also spend time watching television. The design of this study prohibited the determination of whether television use reduces with access to an AVG console. However, because children have been shown to use AVG consoles in a sedentary manner more often and for longer periods of time than in an active manner, it is more likely that playing with an AVG console would be a sedentary alternative to a sedentary activity, rather than an active alternative to a sedentary activity.

This study showed an association between deprivation index and social networking site use, but no other screenbased activities. Results are similar to those of Ogunleye et al. ${ }^{33}$ who reported no evidence of an association between deprivation index and both computer and television use. However, Grund et al. ${ }^{34}$ found an association between socioeconomic status and television viewing among children 5-11 years of age. The variation in results between studies may be due to differences in the age of participants ${ }^{33,35}$ and differences in outcome measures used (i.e., methods of assessing deprivation index and socioeconomic status).

\section{Limitations}

Concerns surrounding sedentary behavior, screen time, and physical activity among children are often triggered by reports on increasing rates of pediatric obesity. As such, much research in this area has examined the relationship between body mass index (BMI) and leisure-time activities. The current study did not measure BMI or ask participants to self-report BMI and therefore cannot draw conclusions on whether those who reported using AVGs had a higher or lower BMI than those who did not report AVG use. BMI was not measured because the main focus of this study was an investigation of screen-based leisure-time activities among children. Furthermore, self-reported height and weight are known not to be reliable among children, and because of the large geographical spread and number of participants in this study, the direct measurement of BMI was not feasible.

As with all self-report physical activity questionnaires there are limitations associated with the Modified Activity Questionnaire for Adolescents. Research has shown that the method of physical activity measurement can affect results, with over-reporting more likely during self-report. Physical activity results of participants in the current study were similar to results of a previous study using the same method in Irish children. ${ }^{5}$ In the current study a more objective form of physical activity measurement was not feasible because of the number of participants being recruited. Particularly for younger participants, the time frame used (recalling activities carried out regularly 1 year ago) may have been too broad. Furthermore, there are limitations associated with deriving MET values from reported physical activities. Some activities reported by children in this study were not available in the compendium of physical activities ${ }^{21}$ and were therefore estimated from similar activities. This may have led to the over- or underestimation of the energy expended by children in this study. Furthermore, when coupling selfreported physical activities with MET levels from the compendium, it is not possible to take the skill or efficiency of the child or environmental conditions into consideration. The questionnaire used in this study was developed with the intent of optimizing the validity of questions and ensuring they were suitable for children approximately 12 years old. The resulting questions asked children to recall physical activities carried out over a longer period of time than sedentary activities, which may limit the comparability of these outcomes. Despite these limitations, the authors believe that the use of a questionnaire to gain information on physical activity levels and sedentary behavior provided some insight into the lifestyle of this population.

Finally, as this study was carried out on children $12.1 \pm 0.6$ years of age in Dublin, results cannot be generalized to children of other ages or those living in other places. However, as a clustered stratified sampling method was used to ensure all geographical areas in Dublin and all levels of deprivation were represented, the results are likely to accurately represent children in fourth class (approximately 12 years old) living in Dublin.

\section{Conclusions}

It was established that exposure to AVGs is high among children $12.1 \pm 0.6$ years of age in Dublin. However, many children did not play AVGs in the 7 days preceding completion of the questionnaire used in this study. Of those who did play games on AVG consoles, more played sedentary games than active games, and sedentary games were played for longer than active games. AVG play was positively correlated with reported time spent watching television and was not associated with MET hours of physical activity. Despite some limitations associated with the self-report nature of outcomes used in this study, more objective means of measurement were not feasible. As this is the first study to examine AVG use in detail and its relationship with other leisure-time activities, we hope that it will add to the body of knowledge in this area. Overall, it can be concluded that children use AVG consoles as a sedentary rather than an active leisure-time activity. This finding may inform studies using AVGs to increase or promote physical activity.

\section{Acknowledgments}

This work was supported by the Irish Research Council for Science Research and Technology (grant G30372).

\section{Author Disclosure Statement}

No competing financial interests exist.

\section{References}

1. Rey-Lopez JP, Vicente-Rodriguez G, Ortega FB, et al. Sedentary patterns and media availability in European adolescents: The HELENA study. Prev Med 2010; 51:50-55.

2. Rideout VJ, Foehr UG, Roberts DF. Generation M2 Media in the Lives of 8-to-18-Year-Olds. Oakland, CA: The Henry J. Kaiser Family Foundation; 2010.

3. American Academy of Pediatrics. Children, adolescents, and television. Pediatrics 2001; 107:423-426. 
4. Trost SG. Objective measurement of physical activity in youth: Current issues, future directions. Exerc Sport Sci Rev 2001; 29:32-36.

5. Hussey J, Gormley J, Bell C. Physical activity in Dublin children aged 7-9 years. Br J Sports Med 2001; 35:268--273.

6. Barnett A, Cerin E, Baranowski T. Active video games for youth: A systematic review. J Phys Act Health 2011; 8:724-737.

7. Biddiss E, Irwin J. Active video games to promote physical activity in children and youth: A systematic review. Arch Pediatr Adolesc Med 2010; 164:664-672.

8. LeBlanc AG, Chaput JP, McFarlane A, et al. Active video games and health indicators in children and youth: A systematic review. PLoS One 2013; 8:e65351.

9. Guinness World Records. Fastest-Selling Gaming Peripheral. www.guinnessworldrecords.com/records-9000/fastestselling-gaming-peripheral/ (accessed November 14, 2014).

10. Nintendo. Consolidated Sales Transition by Region. www .nintendo.co.jp/ir/library/historical_data/pdf/consolidated_ sales_e1406.pdf (accessed November 14, 2014).

11. O'Donovan C, Hussey J. Active video games as a form of exercise and the effect of gaming experience: A preliminary study in healthy young adults. Physiotherapy 2012; 98:205-210

12. O'Donovan C, Roche EF, Hussey J. The energy cost of playing active video games in children with obesity and children of a healthy weight. Pediatr Obes 2014; 9:310-317.

13. Mitre N, Foster RC, Lanningham-Foster L, Levine JA. The energy expenditure of an activity-promoting video game compared to sedentary video games and TV watching. J Pediatr Endocrinol Metab 2011; 24:689-695.

14. Borgers N, De Leeuw E, Hox JJ. Children as respondents in survey research: Cognitive development and response quality. Bull Methodol Sociol 2000; 66:6.

15. Holaday B, Turner-Henson A. Response effects in surveys with school-age children. Nurs Res 1989; 38:248-250.

16. Bell A. Designing and testing questionnaires for children. J Res Nurs 2007; 12:9.

17. Aaron DJ, Kriska AM, Dearwater SR, et al. Reproducibility and validity of an epidemiologic questionnaire to assess past year physical activity in adolescents. Am J Epidemiol 1995; 142:191-201.

18. Huston AC, Wright JC, Marquis J, Green SB. How young children spend their time: Television and other activities. Dev Psychol 1999; 35:912-925.

19. Van Someren MW, Barnard YF, Sandberg JAC. The Think Aloud Method. A Practical Guide to Modelling Cognitive Processes. London: Academic Press; 1994.

20. Kelly A, Telheur C. The National Deprivation Index for Health \& Health Services Research-Update 2013. www.sahru .tcd.ie/services/deprivation/DeprivationFiles/Deprivation Report2013.pdf (accessed November 14, 2014).

21. Ridley K, Ainsworth BE, Olds TS. Development of a compendium of energy expenditures for youth. Int J Behav Nutr Phys Act 2008; 5:45.

22. Arbuckle JL. Full information estimation in the presence of incomplete data. In: Advanced Structural Equation Modelling: Issues and Techniques. London: Psychology Press; 1996; pp 243-279.
23. Strong WB, Malina RM, Blimkie CJ, et al. Evidence based physical activity for school-age youth. J Pediatr 2005; 146: 732-737.

24. Kelly C, Gavin A, Molcho M, Nic Gabhainn S. The Irish Health Behaviours in School-Aged Children (HBSC) Study 2010. Galway, Ireland: NUI Galway; 2012.

25. Troiano RP, Berrigan D, Dodd KW, et al. Physical activity in the United States measured by accelerometer. Med Sci Sports Exerc 2008; 40:181-188.

26. Chung AE, Skinner AC, Steiner MJ, Perrin EM. Physical activity and BMI in a nationally representative sample of children and adolescents. Clin Pediatr (Phila) 2012; 51: 122-129.

27. Serrano-Sanchez JA, Marti-Trujillo S, Lera-Navarro A, et al. Associations between screen time and physical activity among Spanish adolescents. PLoS One 2011; 6: e24453.

28. Halford JC, Gillespie J, Brown V, et al. Effect of television advertisements for foods on food consumption in children. Appetite 2004; 42:221-225.

29. Hamilton MT, Healy GN, Dunstan DW, et al. Too little exercise and too much sitting: Inactivity physiology and the need for new recommendations on sedentary behavior. Curr Cardiovasc Risk Rep 2008; 2:292-298.

30. Chaput JP, Saunders TJ, Mathieu ME, et al. Combined associations between moderate to vigorous physical activity and sedentary behaviour with cardiometabolic risk factors in children. Appl Physiol Nutr Metab 2013; 38:477-483.

31. Grontved A, Ried-Larsen M, Moller NC, et al. Youth screen-time behaviour is associated with cardiovascular risk in young adulthood: The European Youth Heart Study. Eur J Prev Cardiol 2014; 21:49-56.

32. Lanningham-Foster L, Jensen TB, Foster RC, et al. Energy expenditure of sedentary screen time compared with active screen time for children. Pediatrics 2006; 118:e1831e1835.

33. Ogunleye AA, Voss C, Sandercock GR. Prevalence of high screen time in English youth: Association with deprivation and physical activity. J Public Health (Oxf) 2012; 34:46-53.

34. Grund A, Krause H, Siewers M, et al. Is TV viewing an index of physical activity and fitness in overweight and normal weight children? Public Health Nutr 2001; 4:12451251.

35. Melkevik O, Torsheim T, Iannotti RJ, Wold B. Is spending time in screen-based sedentary behaviors associated with less physical activity: A cross national investigation. Int $\mathrm{J}$ Behav Nutr Phys Act 2010; 7:46.

Address correspondence to: Cuisle Forde, PhD

Discipline of Physiotherapy School of Medicine

Trinity Centre for Health Sciences St. James's Hospital Dublin 8, Ireland

E-mail: c.odonovan@tcd.ie 